\title{
Solar panel monitoring and energy prediction for smart solar system
}

Isha M. Shirbhate, Sunita S. Barve

School of Computer Engineering \& Technology, MIT Academy of Engineering, India

\begin{tabular}{l}
\hline \hline Article Info \\
\hline Article history: \\
Received Sep 12, 2018 \\
Revised Apr 5, 2019 \\
Accepted May 5, 2019 \\
\hline
\end{tabular}

\section{Keywords:}

Internet of things (IoT)

Prediction

Solar energy

Solar radiation

Time-series

\begin{abstract}
Solar Energy is established as an alternative source of energy known as renewable energy. In a developing country like India, the perspective of Solar Energy is important, as it supports a limitless source of energy. Monitoring and prediction of photo-voltaic energy generation help to reduce the energy loss and empower to utilize more energy. Solar energy prediction is challenging as it depends on the fluctuating solar radiations and climate conditions. The problem statement is to monitor solar panels and predict energy generation for energy management procedure. In this paper, the Internet of Things and Machine Learning algorithms are used as a powerful tool for developing a smart solar system. The metro-logical data such as humidity, temperature and photovoltaic panel data is used as input to forecast solar power generation. For prediction, we examine time-series of solar energy data with Hidden Markov Model. This model considers the probabilistic correlation between previous values to next value in time-series. Experimental results shows that individual panel dead state is located successfully and timeseries based solar energy prediction emulate the actual power generation.
\end{abstract}

Copyright (C) 2019 Institute of Advanced Engineering and Science. All rights reserved.

\section{Corresponding Author:}

Isha M. Shirbhate,

School of Computer Engineering \& Technology,

MIT Academy of Engineering,

Alandi, Pune, Maharashtra, India.

Email: shirbhate.isha@gmail.com.in

\section{INTRODUCTION}

Solar energy is the best promising natural-renewable energy source for a green future, it has a lesser ecological impact as compared to other usual energy sources. Oil, Coal, Gases are conventional but nonrenewable sources of energy, they will run out very soon if exploited at huge scale. Moreover, the burning of these fossil fuels increases the concentration of greenhouse gases results in global warming [1]. Solar energy does not affect greenhouse atmosphere. Currently, photovoltaic (PV) solar energy characterizes as the thirdlargest source of renewable energy subsequent to hydro and wind [2]. The global investment in photo-voltaic solar systems increased because of transformation in favorable government policies and a reduction in the cost of the PV component. These aspects give rewards for investment in solar systems which is more motivating. PV system faces some problems after installation like dirty surface, damaged connections, and junction box failure results in a reduction in all-over system performance.

IoT is a topology of the linked smart sensors and software that allows the things to assemble and transmit data using the internet. IoT based appliance is very supportive for monitoring the system at cheap cost. Solar panel's energy is affected by environmental conditions. Using sensors we can directly collect details about environmental conditions like humidity and temperature of that location [3-4]. The real-time monitoring system using IoT shows the current status of generated energy, humidity, temperature, a fault at the panel level and details of dead panels [5]. A defect can be detected by collecting and analyzing each sensor's data. Electrical parameters observation generally creates a huge quantity of data. The collected data used by artificial 
intelligence for power forecasting principles [6]. Electrical solar power production is decentralized in nature and it's difficult to monitor a large amount of data [7]. Real-time research of solar system is a very complicated task, as it demands a precise PV emulator that may specifically reproduce the non-linear character of PV cells. It also conflicts realization cost, accuracy, efficiency, complexity, sensitivity with varying environmental conditions [8]. According to author Arun G. Phadkeet [9] by Wide Area Monitoring (WAM), it is possible to assemble dimensions remotely from different places of power systems. Monitoring PV panels perform supervision along with fault recognition. The fault finding process assists for the recognizing diagnosis of the solar plant system, which depends on the study of voltage and current factors anticipated from monitored data of a PV [10]. This facilities improves WAM to modern energy system protection. The proper management meets a new scheme which gives an advance result of the adaptive system.

To develop a smart solar plant system, it is essential to get accurate PV power forecasting results for efficient energy management. The messy nature of ecological conditions and the surprising weather circumstances makes accurate solar power prediction enormously difficult [11]. Prediction of solar power is straightforwardly connected to the weather predictions. To make well-organized consumption of energy system, it is helpful to forecast the reports of solar energy generation. The exact forecasting of solar irradiance digression enhances the quality of progression with civilizing PV energy supervision circumstances [12]. Maximum Power Point Tracking (MPPT) for solar systems assist prediction by measuring and estimating voltage and current. The Adaptive Neuro-Fuzzy (ANFIS) model utilizes PV cells temperature and solar radiation with a wavelet denoising model to obtain filtered constraints. MATLAB is easy to revise the processing of the MPPT [13].

The problem statement of the proposed system is to monitor solar panels and predict the future energy generation using Hidden Markov Model algorithm for energy management in IoT base environments. The objectives of the proposed system are as follows: to develop a real-time solar panel monitoring system for more energy utilization; and to develop a prediction model for solar energy management procedure.

\section{SYSTEM ARCHITECTURE}

The proposed smart solar system starts with monitoring panel level PV plant. The solar energy monitoring system is implemented by using IOT. Figure 1 shows the system architecture of a proposed system. We can observe the solar panel and multiple sensors and PV panels are connected with the middleware electronic board that is Raspberry Pi 3. That board is a central observing structure which collects data from sensors. A temperature and humidity of the location are sensed by sensors and power values are sensed by PV panel. Monitoring system displays the current status of temperature, humidity and generating energy power at certain time intervals. The system displays the entire information on the web pages and subsequently, it stores data on the cloud.

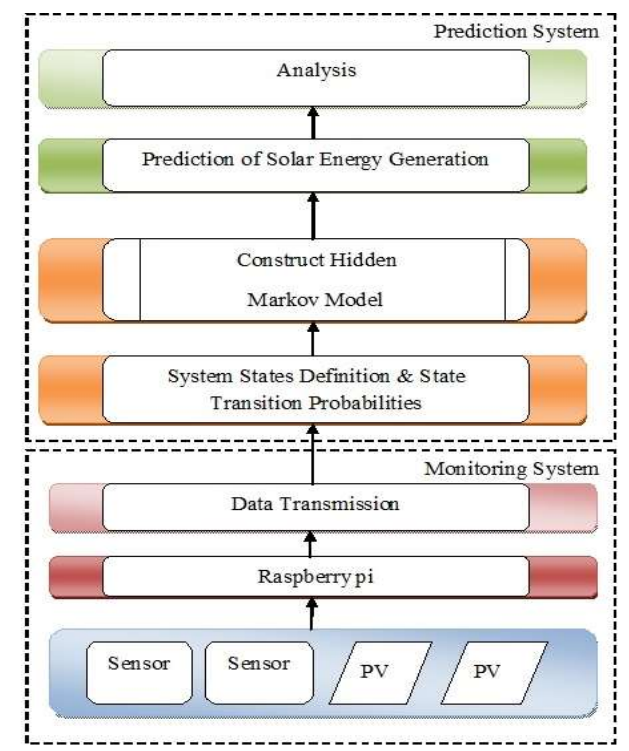

Figure 1. Architecture diagram of proposed system 
Prediction system works on historical data of solar power collected by monitoring system. To make advance adaptive forecast system we apply a Hidden Markov Model (HMM) which works as time-series prediction. The system states definition and transition probabilities for constructing HMM. The proposed method gives an accurate prediction of solar energy generation.

\section{RESEARCH METHOD}

\subsection{Monitoring system}

The monitoring system measure and display the collected data by sensors and solar panels. We monitored temperature, humidity and generating solar power using smart devices. It is essential for a monitoring system that the customers must know the potential and practical boundaries of devices, operational deviations and objective of data analysis. As we know python is a largely used, advanced, interpreted, highlevel, dynamic programming language, so we use python programming to complete this task. Monitoring constraints are then uploaded on the cloud using a python program. Flask works as a light-weight framework, which is written in Python. The Flask uses stretchy cast off to read the sensor values. We consider the DHT11 sensor that shows temperature with humidity as weather information from real-time situations. The system can work on the multiple solar panels which had the heterogeneous configuration.

\subsection{Prediction system}

In the proposed system, we work on HMM for finding the most probable time-series. The time-series prediction is calculated by estimating probability of state transition and observation sequences for the predetermined state which gives precision in prediction. This method takes historical records of power as a input. Markov chain property says that the possibility of every subsequent state depends just on earlier state $[14,15]$. The HMM have $N$ states, known as $s_{1}, s_{2}, \ldots, s_{N}$ where discrete time-period $t=0,1, \ldots$ On each timeperiod, the system is in the state which is freely available called $N$ states. These states with time $t$ is identify like $Q_{t}$, by $Q_{t} \in\left\{s_{1}, s_{2}, \ldots, s_{N}\right\}$. At each and every time-period, the state $Q_{t}$ form, one resulting symbol according to Observation and Emission Probabilities allocation.

We find hidden and observed states called as a state probability vector $\boldsymbol{\Pi}$, inter-state transition probability matrix $\boldsymbol{A}$ and emission probability matrix $\boldsymbol{B}$. Markov chain can translate calculated data to the various states, so the series of energy generation values is translated into the number of states.

Therefore, State transition probabilities are $\left\{s_{1}, s_{2}, \ldots, s_{N}\right\}$

It signifies probability of one state to another state as a state transition probability matrix

$$
A=\left(a_{i j}\right), a_{i j}=\mathrm{P}\left(s_{i} \mid s_{j}\right)
$$

Here, $i$ represents a number of rows and $j$ number of columns. The observation probability allocation signify for state $j$ is $V m, m=1, \ldots . M$ in the state $s_{j}$

$$
B=\left(b_{i}\left(v_{m}\right)\right), b i\left(v_{m}\right)=\mathrm{P}\left(v_{m} \mid s_{i}\right)
$$

As quantity of symbols are two thus $m=2$ With vector initial probability matrix

$$
\Pi=\left(\Pi_{i}\right), \Pi_{\mathrm{i}}=\mathrm{P}\left(s_{i}\right)
$$

At last, Model is shown by $\lambda=(A, B, \Pi)$.

The purpose of energy prediction is examining a Hidden Markov Model by considering observed time- series. Maximum likelihood estimation method estimate $\lambda^{*}$ which maximizes a likelihood of training series, $O T s=\left\{O T s_{t}\right\}_{t=1}^{T}$ for improvement in $\mathrm{P}(O T s \mid \lambda)$. Autonomous and supremely spotted samples of, OTs $=\left\{O T s_{t}\right\}_{t=1}^{T}$ are anxious from the probability allocation $\mathrm{P}($ OTs $\mid \lambda)$. The objective is to get a value of $\lambda$ which originates $O T s_{t}$ to $\mathrm{P}(O T s \mid \lambda)$ as likely as probable.

$$
O T s_{t} \cong \mathrm{P}(O T s \mid \lambda)
$$

The probability variation method is precise with identified $\lambda=(A, B, \Pi)$. Recalculate $\lambda$ for the entire steps with likelihood probability of frequency of state $s_{j}$ following state $s_{i}$. This variation step is reiterate till convergence for the time-interval of which $\mathrm{P}($ OTs $\mid \lambda)$ not reduces.

Int. J. of Adv. in Appl. Sci. Vol. 8, No. 2, June 2019: 136 - 142 


\begin{tabular}{|c|c|}
\hline Steps & Algorithm \\
\hline 1: & Observe Time-Series in Sequence \\
\hline & OTs $=\left\{0^{\prime} s, 1^{\prime} s\right\}$ \\
\hline 2: & $\begin{array}{l}\text { Named as different observation symbols for states } N \\
\qquad V=\{0,1\}\end{array}$ \\
\hline 3: & $\begin{array}{l}\text { Calculate approximately values of Markov model to maximize probability } \mathrm{P}(\text { OTs } \mid \lambda) \\
\qquad \lambda=(A, B, \Pi)\end{array}$ \\
\hline 4: & Predict Energy generation depends on past observation time-series \\
\hline & $\mathrm{P}($ OTs, $1 \mid \lambda)<\mathrm{P}($ OTs, $0 \mid \lambda)$ then $S(t+1)=0$ \\
\hline & $\mathrm{P}($ OTs, $1 \mid \lambda) \geq \mathrm{P}($ OTs, $0 \mid \lambda)$ then $S(t+1)=1$ \\
\hline 5: & $\begin{array}{l}\text { Produce predicted observation time-series for } t=T+1 \text { to } 2 T \\
P O T S=\{O T s\}^{2 T}\end{array}$ \\
\hline
\end{tabular}

The algorithm initially examines all the records from the dataset and spites every characteristic. At step 2, it mentions each attribute as a unique symbol i.e. $0^{\prime} s$ and $1^{\prime} s$. The sampling approach work with the help of HMM model in step 3. Then next we do prediction of observation time-series of moving ratio of input. Lastly, in conclusion step 5 gives the prediction output. This process is called as an observable Markov Model. It gives a set of number of states at the specific time period, where every state communicates to the next state [16]. Thus the presented model seems preventive for several problems. We can expand the idea of a Markov Model to embrace the sets while observation sequence is a probabilistic method of states. HMM is a vital version of Markov method, yet HMM is a self-governing procedure. Here, states communicate to a group of same probability allocation of observation. While working on this algorithm we found two challenging problems that are mention.

\subsubsection{Likelihood problem}

The first problem is to calculate the likelihood sequence of a distinct observation sequence that is a new observation sequence and a set of models. It finds a model which gives the best sequence. The best possible sequence linked with known observations using state sequence and Observed Time-Series

$$
\mathrm{P}(\text { OTs } \mid Q)=\Pi \mathrm{P}\left(\text { OTs } \mid s_{i}\right) \times \Pi \mathrm{P}\left(s_{i} \mid s_{i-1}\right)
$$

We used an efficient algorithm called the forward algorithm. The forward algorithm is a kind of dynamic programming algorithm. It computes the observation probability by summing over the probabilities of all possible hidden-state paths that could generate the observation sequence.

\subsubsection{Decoding problem}

HMM model, which contains hidden variables, the challenge is to determine which sequence is the underlying source of some sequence of observations. It is called the decoding task. The decoder finds the besthidden sequence, this problem solved by the Viterbi algorithm. It is possible to alter the model constraints $\lambda=$ $(A, B, \Pi)$ for maximize $\mathrm{P}($ OTs $\mid \lambda)$. The Viterbi Algorithm score finest from the a particular path at initial time $t$ observations, trim with state $s_{i}$, presented as:

$$
\delta(i)=\max \mathrm{P}\left(s_{1}, s_{2}, \ldots, s_{N}=i, \text { OTs } \mid \lambda\right)
$$

\section{RESULTS AND DISCUSSION}

The results are split up into three parts. First part is a performance evaluation of the monitoring system. The second part is an analysis and comparison of the prediction model and the third part is a case-study on a proposed method.

\subsection{A solar plant monitoring system}

The system shows a status dashboard of monitoring panels with energy generation level. It monitors the temperature in degree celsius and humidity in the percentage. Solar energy differs by environmental seasons [3], Figure 2 shows graphs for hourly temperature and solar energy generation in sunny, cloudy and rainy days. The system represents graphs for analyzing the performance in a standard method to provide the understandable view of information to the users. The dashboard is accessible to the users from anywhere and anyplace. If the one-time connection to the server is successful, the status of the system is constantly passed to the web server for monitoring the parameters of solar Power Conditioning Unit (PCU). We can examine the intense values generated by solar PCU. The system is able to analyze past average ratio of energy generation monthly and yearly. In addition, the system also forecasts the panel's dead state and the error finding. 


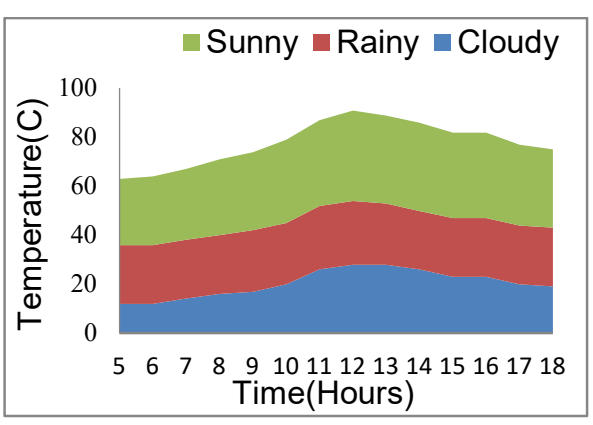

(a)

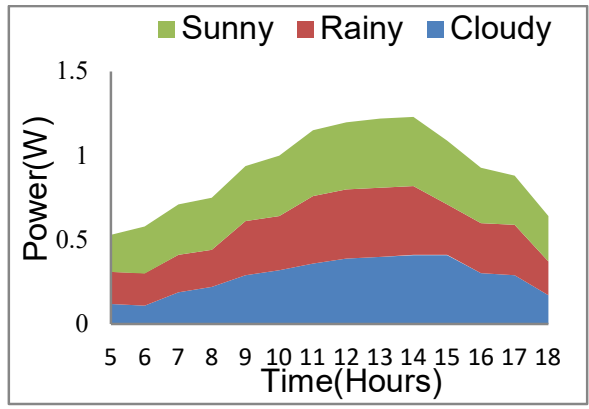

(b)

Figure 2. (a) Temperature and (b) Power in different weather conditions

\subsection{Solar energy prediction}

We identified that the consequences of prediction are affected by the environmental situations and location of PV plant [6]. In the prediction model, we used an advanced Hidden Markov Model to predict solar energy generation. Considering the real-time statistics measured by the monitoring system, we get better results. Following Table 1 shows the actual values and predicted values, for few hourly sample cases.

Accurateness of prediction is calculated with help of Root Mean Square Error (RMSE) and Mean Absolute Percentage Error (MAPE). We compare the proposed algorithm with Linear Regression [17] as shown in Table 2. If rate among predicted and actual values are close to zero by RMSE, then we find betterquality output at low error. Figure 3 and Figure 4 show day wise and month-wise, actual with predicted PV power generation for different environmental conditions.

Table 1. Different sample cases on hourly data

\begin{tabular}{ccccc}
\hline Hour & Observation Seq & States & Actual Output & Proposed Model \\
\hline 1 & $1-0-1-\ldots \ldots . .0$ & 2 & 1.44 & 1.56 \\
2 & $0-1-0-\ldots \ldots . .1$ & 2 & 1.50 & 1.57 \\
3 & $1-1-0-\ldots \ldots . .1$ & 2 & 1.82 & 1.67 \\
4 & $0-1-1-\ldots \ldots \ldots . .0$ & 2 & 1.55 & 1.65 \\
\hline
\end{tabular}

Table 2. Comparison between proposed method and another prediction method

\begin{tabular}{cccc}
\hline Sr. No. & Method & RMSE & MAPE \\
\hline 1 & Proposed Method & 0.642 & 8.442 \\
2 & Linear Regression & 1.501 & 13.440 \\
\hline
\end{tabular}
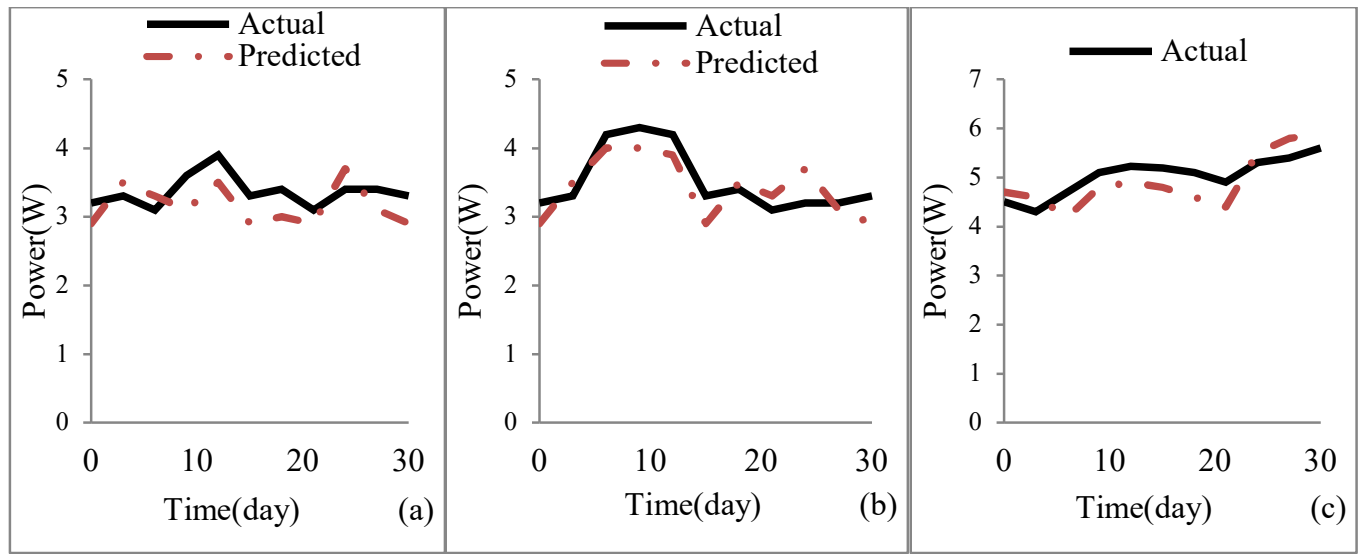

Figure 3. Actual and predicted PV power generation in (a) Cloudy, (b) Rainy and (c) Sunny month 


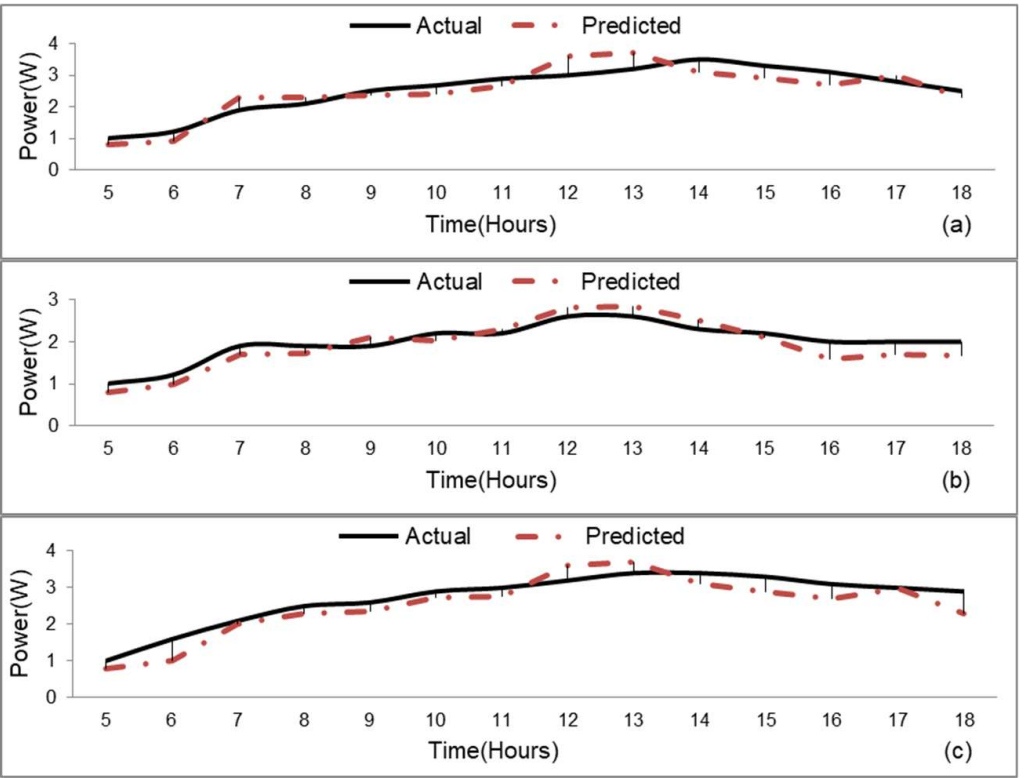

Figure 4. Actual and predicted PV power generation for (a) Cloudy, (b) Rainy and (c) Sunny day

\subsection{Case study}

The proposed methodology is tested on a database of the large-scale system, which is located at MIT Academy of Engineering, Alandi, Pune campus as shown in Figure 5. There are six distributed solar plants, one single panel is with $315 \mathrm{watt}$ peak capacity and whole solar system having the $435 \mathrm{~kW}$ capacity. The system added with irradiance sensor, temperature sensor and wind speed sensor for monitoring the weather situations. We consider the hourly power data of one plant to predict the solar power. Figure 6 shows the actual and predicted power generation of the solar plant.

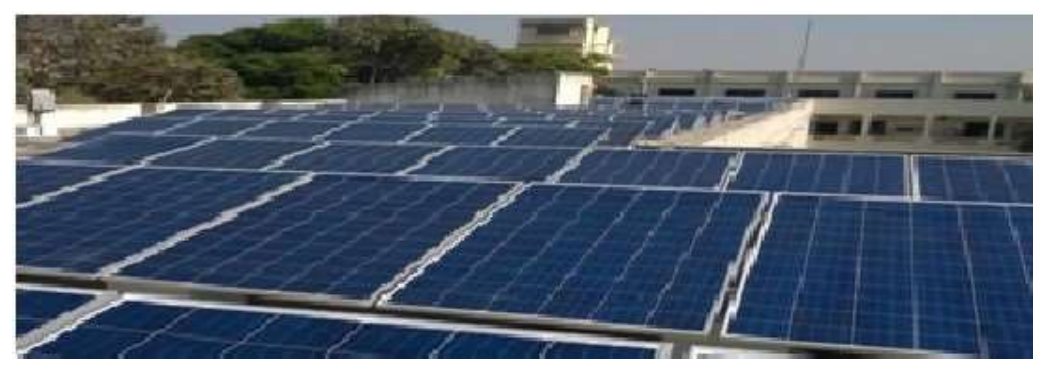

Figure 5. Large-scale solar plant at MIT Academy of Engineering, Alandi

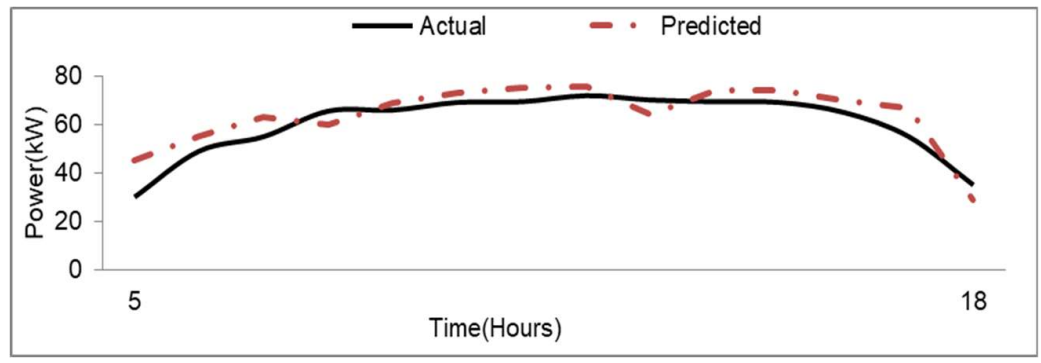

Figure 6. Actual and predicted PV power generation of large-scale solar plant 


\section{CONCLUSION}

Solar energy is trustworthy and sustainable hence the utilization of this smart solar system is reliable, adequate and cost-efficient. The proposed smart solar system comprises both Monitoring and Prediction. We have used the Internet of Things (IoT) for monitoring by considering parameters like Temperature, Humidity and Solar Power. Internet of Things is providing practically expert methods that offers required outcome. The designed system monitors panel level PCU and anticipates the error findings. We can analyze the weekly or monthly performance of panels. On the other hand, Prediction uses Hidden Markov Model for forecasting the solar power. We have developed an hourly prediction system. Considering historical records, the proposed model is able to predict accurate power generation in time- series method. Numerical results show that the proposed model achieves better prediction accuracy than the simple Linear Regression model.

\section{REFERENCES}

[1] I. Gherboudj and H. Ghedira, "Assessment of solar energy potential over the United Arab Emirates using remote sensing and weather forecast data," Renewable and Sustainable Energy Reviews, vol. 55:1210-1224, 2016.

[2] Moreno-Garcia, E. Palacios-Garcia, V. Pallares-Lopez, I. Santiago, M. Gonzalez-Redondo, M. Varo Martinez, and R. Real-Calvo, "Real-time monitoring system for a utility-scale photovoltaic power plant," Sensors, vol. 16(6), p. 770, 2016.

[3] S. R. Madeti and S. N. Singh, "Monitoring system for photovoltaic plants: A review," Renewable and Sustainable Energy Reviews, vol. 67, pp. 1180-1207, 2017.

[4] B. Ando, S. Baglio, A. Pistorio, G. M. Tina, and C. Ventura, "Sentinella: Smart monitoring of photovoltaic systems at panel level," IEEE Transactions on Instrumentation and Measurement, vol. 64(8), pp. 2188-2199, 2015.

[5] F. O. Hocaoglu and F. Serttas, "A novel hybrid (Mycielski-Markov) model for hourly solar radiation forecasting," Renewable Energy, vol. 108, pp. 635-643, 2017.

[6] S. Daliento, A. Chouder, P. Guerriero, A. M. Pavan, A. Mellit, R. Moeini, and P. Tricoli, "Monitoring, diagnosis, and power forecasting for photovoltaic fields: A review," International Journal of Photoenergy, 2017.

[7] C. Stegner, M. Dalsass, P. Luchscheider, and C. J. Brabec, "Monitoring and assessment of PV generation based on a combination of smart metering and thermographic measurement," Solar Energy, vol. 163, pp. 16-24, 2018.

[8] S. Senthilkumar, G. P. C., "Hidden Markov Model based channel selection framework for cognitive radio network," Computers and Electrical Engineering, vol. 65, pp. 516-526, 2018.

[9] A. G. Phadke, P. Wall, L. Ding, and V. Terzija, "Improving the performance of power system protection using wide area monitoring systems," Journal of Modern Power Systems and Clean Energy, vol. 4(3), pp. 319-331, 2016.

[10] S. Silvestre, L. Mora-Lopez, S. Kichou, F. Sanchez-Pacheco, and M. Dominguez-Pumar, "Remote supervision and fault detection on OPC monitored PV systems," Solar Energy, vol. 137, pp. 424-433, 2016.

[11] C.Wan, J. Zhao, S. Member, and Y. Song, "Photovoltaic and solar power forecasting for smart grid energy management," Journal of Power and Energy Systems, vol. 1(4), pp. 38-46, 2015.

[12] S. Mohanty, P. K. Patra, S. S. Sahoo, and A. Mohanty, "Forecasting of solar energy with application for a growing economy like India: Survey and implication," Renewable and Sustainable Energy Reviews, vol. 78, pp. 539-553, 2017.

[13] A. Chikh and A. Chandra, "An optimal maximum power point tracking algorithm for PV systems with climatic parameters estimation," IEEE Transactions on Sustainable Energy, vol. 6(2), pp. 644-652, 2015.

[14] M. J. Sanjari and H. B. Gooi, "Probabilistic forecast of pv power generation based on higher order markov chain," IEEE Transactions on Power Systems, vol. 32(4), pp. 2942-2952, 2017.

[15] S. S. Barve, "Dynamic channel selection and routing through reinforcement learning in cognitive radio networks," IEEE International Conference on Computational Intelligence and Computing Research, 2012.

[16] W. J. I. Li and Jinbo Pedrycz, "Multivariate time series anomaly detection: A framework of hidden markov models," Applied Soft Computing Journal, vol. 60(27), pp. 229-240, 2017.

[17] N. Sharma, P. Sharma, D. Irwin, and P. Shenoy, "Predicting solar generation from weather forecasts using machine learning," Smart Grid Communications (SmartGridComm), 2011 IEEE International Conference, pp. 528-533, 2011. 